\title{
Effect of spatial resolution on estimating the Sun's magnetic flux
}

\author{
N. A. Krivova and S. K. Solanki
}

\author{
Max Planck Institute for Aeronomy, 37191 Katlenburg-Lindau, Germany \\ e-mail: solanki@linmpi.mpg.de
}

Received 29 August 2003 / Accepted 14 December 2003

\begin{abstract}
A critical question related to a possible secular trend in the Sun's total magnetic flux and consequently in solar irradiance is the total amount of magnetic flux present on the Sun and how it is distributed between active regions and the quiet Sun. NSO/Kitt Peak synoptic charts have in the past been used to estimate the total flux and the fraction of the flux in active regions and in the quiet Sun. Since a single pixel of these synoptic charts is much bigger than individual small-scale magnetic elements and opposite polarities may be present within the same pixel, some magnetic flux escaped notice. Here we estimate the fraction of the magnetic flux escaping detection in Kitt Peak synoptic charts. By artificially reducing the spatial resolution of MDI full-disc and high-resolution magnetograms we study the influence of the resolution on the measured total magnetic flux. Noise in the data poses the main difficulty to this approach and is carefully studied. It is concluded that at least half of the magnetic flux in the quiet Sun remains undetected in Kitt Peak synoptic charts and that the total flux present on the solar surface at maxima of activity is around twice the flux present at activity minima.
\end{abstract}

Key words. Sun: activity - Sun: faculae - Sun: magnetic fields - Sun: solar-terrestrial relations - Sun: sunspots

\section{Introduction}

The total solar irradiance is now known to vary at all observable time-scales (Willson \& Hudson 1988, 1991; Fröhlich 2000). This variability, so weak that it escaped notice up to nearly the end of the 20th century, may affect the Earth's climate (e.g., the volume edited by Wilson 2000). Variations in the radiative output of the Sun are directly allied to changes in the amount and distribution of the solar surface magnetic field (Fligge et al. 2000a,b; Krivova et al. 2003). The distribution and evolution of active regions on the solar disc provides important contributions to solar irradiance changes on time-scales of days to the length of the magnetic activity cycle.

Of even greater importance for the Earth is a possible secular trend in the irradiance variability, caused by secular changes in the quiet-Sun magnetic field (Solanki et al. 2000; Solanki 2002). It is expected that since the Maunder minimum a significant amount of background, or "quiet Sun" flux has been built up (Solanki et al. 2002, and references therein). A knowledge of the total amount of flux present at sunspot minimum can thus provide an upper limit on the irradiance increase since the Maunder minimum (by assuming that the Sun was devoid of magnetic flux at the end of the Maunder minimum).

In an important study, Harvey (1994) examined the evolution of the magnetic field in active regions and in the quiet Sun over solar cycles 21 and 22 using NSO/Kitt Peak (KP) synoptic

Send offprint requests to: N. A. Krivova,

e-mail: natalie@linmpi.mpg.de charts. She found that the magnetic field in the quiet Sun dominates during a solar activity minimum and shows little variation on the time-scale of a solar cycle. At activity maximum active regions contain most of the Sun's magnetic flux, with the magnetic flux in active regions, $\Phi_{\mathrm{AR}}$, exceeding by up to a factor of 3 the flux in the quiet Sun, $\Phi_{\mathrm{QS}}$. We expect this ratio to be too large for the following reason: the magnetic field outside active regions is highly non-uniform, with magnetic elements of opposite polarity often being located close together, typically at scales smaller than the size of a pixel in the KP synoptic charts ( $1^{\circ}$ in longitude and 0.011 in sine latitude). This leads to an apparent cancellation of the flux within a pixel of a Kitt Peak synoptic chart and thus to an underestimate of the total magnetic flux. We expect this effect to be particularly strong for the quiet Sun, since active regions often have larger areas of a single polarity.

Here, we study the influence of the spatial resolution of magnetograms or synoptic maps on the measured magnetic flux. We use full-disc and high-resolution magnetograms recorded by MDI (Michelson Doppler Interferometer; Scherrer et al. 1995) on board SOHO to estimate the total magnetic flux of the Sun. The reason for employing MDI data is the fact that, in contrast to ground-based magnetograms, they are not affected by seeing, but have constant and well-defined spatial resolution. The employed data and method of analysis are described in Sect. 2. The results of the analysis are described and discussed in Sect. 3. Our conclusions are presented in Sect. 4. 


\section{Data and method}

We first consider MDI full disc magnetograms having spatial resolution (2 pixels) of $4 "$ (Scherrer et al. 1995) and create synoptic charts therewith. Two Carrington Rotations in solar cycle 23 - 1915 (October-November 1996, when the Sun was very quiet) and 1975 (April-May 2001, near the maximum of solar activity) - have been analysed. In its simplest form the analysis consists of degrading the resolution of the synoptic charts created from the MDI magnetograms step by step until the resolution of the Kitt Peak synoptic charts employed by Harvey (1994) is reached and comparing the unsigned flux in the original and degraded synoptic charts. The main complication for the interpretation of the results obtained from this procedure is produced by the noise in the data.

The $1 \sigma$ noise in the original MDI magnetograms obtained with a 1-min cadence is typically about $20 \mathrm{G}$ (Scherrer et al. 1995; see also Ortiz et al. 2002 and Sect. 3.2 of the present Paper). This is comparable to the magnetic signal of quiet network features. Moreover, the effect of the noise is very similar to that of the signal from a mixed polarity distribution of smallscale elements: since the noise contribution to different pixels is largely uncorrelated, binning pixels dominated by noise, i.e. pixels possessing apparent signals of opposite signs, leads to a rapid drop of the apparent net signal. If not taken into account, noise can lead to an overestimate of the effects of spatial smearing. To reduce the noise level, we average over 5, 20 or 56 consecutive individual magnetograms (which reduces the noise to $\sigma \approx 8.9 \mathrm{G}, 4.5 \mathrm{G}$ and $2.7 \mathrm{G}$, respectively). When averaging, we take solar differential rotation into account by rotating all magnetograms in a set to the time of a fiducial magnetogram before averaging. If the absolute signal in a pixel is less than $1 \sigma$ we set it to 0 . Since in this case a considerable amount of noise is identified as magnetic signal, we also use $2 \sigma$ and $3 \sigma$ thresholds. Choosing a threshold is a tradeoff between the number of pixels affected by noise (lower for higher thresholds) and the fraction of the Sun's flux lying below the threshold (higher for higher thresholds). It is clear that a significant amount of real signal vanishes too, in particular for the higher thresholds and the 1- and 5-min averages.

The low-noise 20- and 56-min averages can, unfortunately, not be produced for the period of minimum activity, since sufficiently long bursts of magnetograms were not recorded at that time. Another problem with these low-noise maps is that magnetic features evolve in the course of an hour and their peculiar motion can transport a given magnetic feature out of a given pixel in this time. Note that the size of a pixel of the MDI fulldisc magnetograms corresponds to the typical size of a granule, while the typical lifetime of a granule is short compared to the integration time of $1 \mathrm{~h}$. Since the flux redistributes itself in the intergranular lanes when new granules are formed there are ample opportunities for the magnetic flux to move to a neighbouring pixel. This means that in these averaged magnetograms the magnetic signal is smeared. This smearing causes it to be further reduced, partly because some of it then falls below the noise limit, partly due to cancellation with opposite polarity flux in the neighbouring pixels. The obtained synoptic maps are also corrected for the fact that the magnetograms measure only the longitudinal component of the magnetic field while in reality it is mainly vertical, outside of sunspots. This is achieved by dividing the absolute value of the flux in each pixel by its corresponding $\mu$-value $(\mu=\cos \theta$, where $\theta$ is the heliocentric angle).

Since active regions are in general more uniform than the quiet Sun network (in the sense that the former contain larger unipolar patches of magnetic field), the effect of the spatial resolution should be weaker for the former. Therefore, we consider not only the Sun as a whole but also the quiet Sun and the active regions separately. To isolate active regions, the technique proposed by Harvey (1994) is used. Every pixel in the synoptic chart is analysed for the amplitude of the variation of its magnetic field with respect to the neighbouring pixels. For this, the absolute value of the magnetic field in this pixel is compared to the absolute value of the field in every other pixel of a box of $41 \times 41$ pixels centred at the pixel of interest. The root-mean-square-differences are calculated for all pixels and then smoothed over 20 pixels to eliminate patchiness. If the difference exceeds $2000 \mathrm{G}$ then the pixel is considered to belong to an active region. About 10\% of all pixels during CR 1975 and less than $0.5 \%$ during CR 1915 fall within active regions according to this definition. The left column of Fig. 1 shows the three resulting maps (56-min averages, noise threshold at $1 \sigma$ ) for CR 1975: the total flux (top panel), active regions (middle) and the quiet Sun (bottom). On the whole, the technique of Harvey (1994) separates active regions and network (as judged by eye) quite well from each other. A few isolated patches of active network (decaying active regions) are identified as active regions. We do not expect that the few such possible misidentifications significantly affect the following results and hence do not correct for them manually.

To compare the synoptic charts obtained from MDI data with those having KP synoptic chart resolution, we reduce the resolution of the former by binning subarrays of $9 \times 6$ pixels together (see Harvey 1994; Scherrer et al. 1995). Hereafter, maps or magnetograms obtained from MDI data in this way will be referred to as "KP, SC". Active regions are isolated in these new, low-resolution maps too and the threshold between the active and quite Sun is adjusted to give the same relative areas for both components as in the high resolution synoptic charts. The resulting "KP, SC" maps obtained from 56-min MDI averages for CR 1975 are shown in the right column of Fig. 1. The results of the comparison of the MDI and "KP, SC" charts are presented in Sects. 3.1 and 3.2.

In Sect. 3.3 we consider MDI magnetograms obtained in the high-resolution mode (spatial resolution of $1.2^{\prime \prime}$ ). As for the synoptic charts, we reduce the noise by averaging 20 sequential individual magnetograms $(\sigma \approx 4.5 \mathrm{G})$. The peculiar motion of magnetic elements becomes more significant in the high-resolution mode, so that we found $20 \mathrm{~min}$ to be the maximum interval before smearing of features begins to be important. E.g. 56-min integrated high-resolution magnetograms exhibit distinct signs of smearing. 

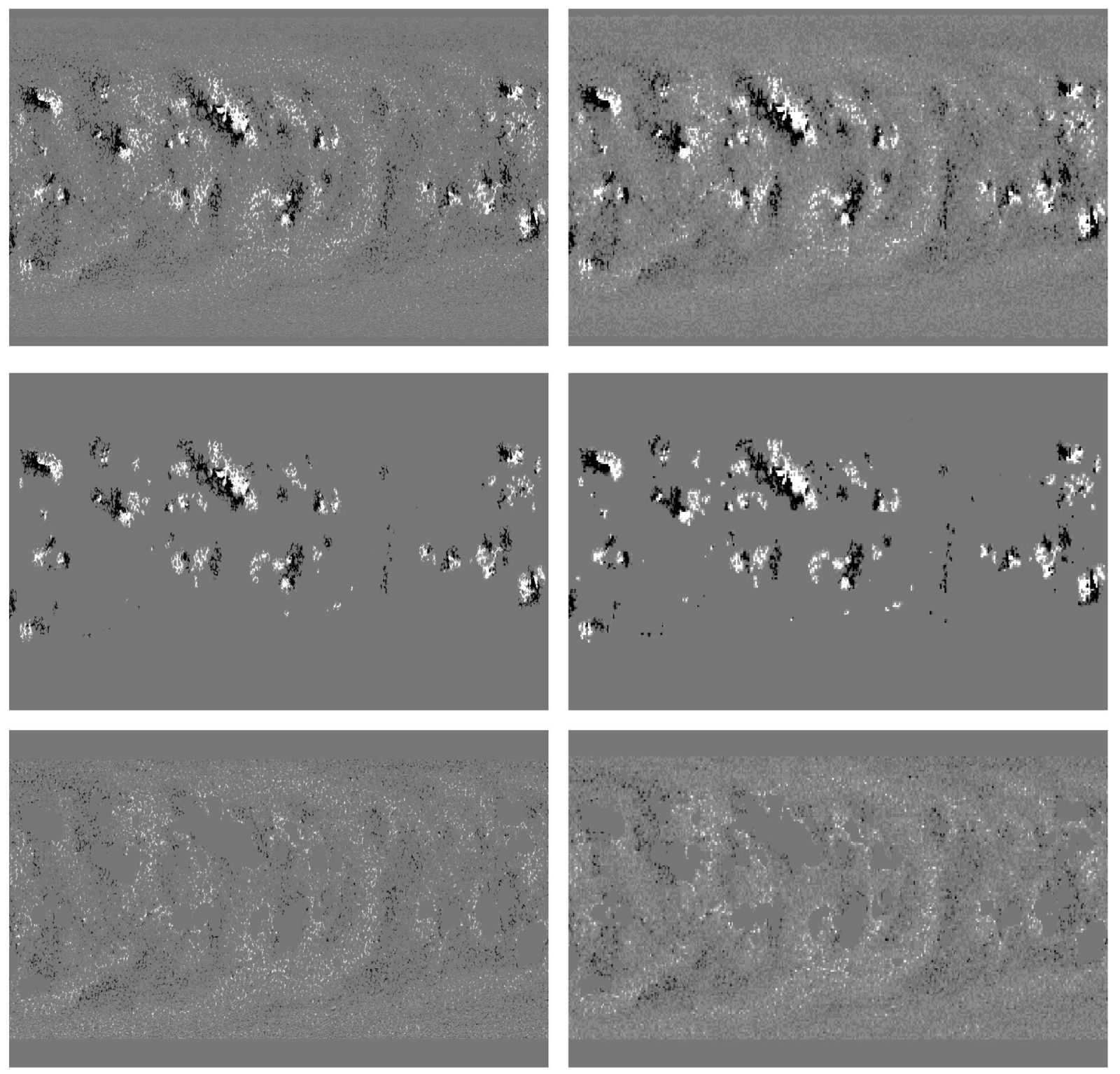

Fig. 1. Synoptic charts for the period 9th April-6th May 2001 created from MDI full disc magnetograms averaged over 56 min. Left: original resolution; right: resolution reduced to that of the KP synoptic charts. Top panels: the total magnetic flux above $1 \sigma$; middle: only active regions; bottom: only the quiet Sun. Dark and light mark negative and positive polarities, respectively, while grey indicates zero flux.

\section{Results}

\subsection{Full disc MDI magnetograms and synoptic charts}

The ratio of the total magnetic flux obtained from synoptic charts with the original MDI resolution to that from the charts with degraded resolution ("KP, SC") is listed in Table 1. The results for different integration times and noise thresholds (1, 2 and $3 \sigma$ ) for the two considered Carrington Rotations are tabulated. Thresholding at $2 \sigma$ and $3 \sigma(40$ and $60 \mathrm{G}$ ) for 1-min maps carries very little information, since practically all small-scale magnetic elements are eliminated in this way. The ratios resulting for these cases have therefore not been included in Table 1. Note that for CR 1915, when the Sun was very quiet, the results for the whole Sun and only the quiet Sun are almost identical, since less than $0.5 \%$ of the solar surface was covered by active regions at that time.
It is immediately obvious that the spatial resolution is rather unimportant for determining the total magnetic flux of active regions: the "missing" flux (i.e. the flux not seen in the Kitt Peak synoptic charts, but present in the MDI magnetograms) is less than $5 \%$ of the total, whereas it is far more important for the quiet Sun (although the situation is more intricate there, with the fraction of "missing" flux depending on the threshold and the integration time of the magnetogram).

The situation is illustrated by Fig. 2. Here, we have cut the CR 1975 synoptic chart obtained from magnetograms averaged over $56 \mathrm{~min}$ into 28 vertical strips, each corresponding to one day. We have then found the ratio $\Phi_{\mathrm{MDI}} / \Phi_{\mathrm{KP}}$, SC for the total flux, quiet Sun and active regions for each strip and plotted them versus the corresponding $\Phi_{\mathrm{MDI}}$. The ratios for the total flux are denoted by asterisks, whereas diamonds and plus signs represent the quiet and active Sun, respectively. Ratios 
Table 1. Magnetic flux, $\Phi_{\mathrm{MDI}}$, of synoptic maps (CR 1915 and CR 1975) created from MDI full-disc magnetograms relative to the flux that would be measured for these maps with the KP synoptic chart resolution, $\Phi_{\mathrm{KP}}, \mathrm{sc}$. Total flux (AR+QS) as well as flux from active regions (AR) and quiet Sun (QS) are listed separately. The synoptic charts covering a full Carrington rotation each were made using original 1-min magnetograms, as well as 5-, 20- and 56-min averages, if a sufficient number of magnetograms was available. Signal below $1 \sigma, 2 \sigma$ or $3 \sigma$ was assumed to be noise in the three considered cases, respectively.

\begin{tabular}{|c|c|c|c|c|c|c|c|c|c|c|}
\hline & \multirow{2}{*}{$\begin{array}{c}1-\min \\
(\sigma=20 \mathrm{G}) \\
1 \sigma\end{array}$} & \multicolumn{3}{|c|}{$\begin{array}{c}5-\min \\
(\sigma=8.9 \mathrm{G})\end{array}$} & \multicolumn{3}{|c|}{$\begin{array}{c}20-\min \\
(\sigma=4.5 \mathrm{G})\end{array}$} & \multicolumn{3}{|c|}{$\begin{array}{c}\text { 56-min } \\
(\sigma=2.7 \mathrm{G})\end{array}$} \\
\hline & & $1 \sigma$ & $2 \sigma$ & $3 \sigma$ & $1 \sigma$ & $2 \sigma$ & $3 \sigma$ & $1 \sigma$ & $2 \sigma$ & $3 \sigma$ \\
\hline \multicolumn{11}{|c|}{ CR 1975} \\
\hline $\mathrm{AR}+\mathrm{QS}$ & 1.38 & 1.30 & 1.12 & 1.05 & 1.25 & 1.15 & 1.09 & 1.22 & 1.15 & 1.10 \\
\hline AR & 1.05 & 1.04 & 1.03 & 1.03 & 1.05 & 1.04 & 1.03 & 1.04 & 1.04 & 1.03 \\
\hline QS & 1.97 & 1.75 & 1.31 & 1.10 & 1.60 & 1.36 & 1.21 & 1.50 & 1.35 & 1.23 \\
\hline \multicolumn{11}{|c|}{ CR 1915} \\
\hline $\mathrm{AR}+\mathrm{QS}$ & 2.04 & 1.73 & 1.16 & 1.03 & & & & & & \\
\hline AR & 1.00 & 1.04 & 1.03 & 1.03 & & & & & & \\
\hline QS & 2.08 & 1.77 & 1.17 & 1.03 & & & & & & \\
\hline
\end{tabular}

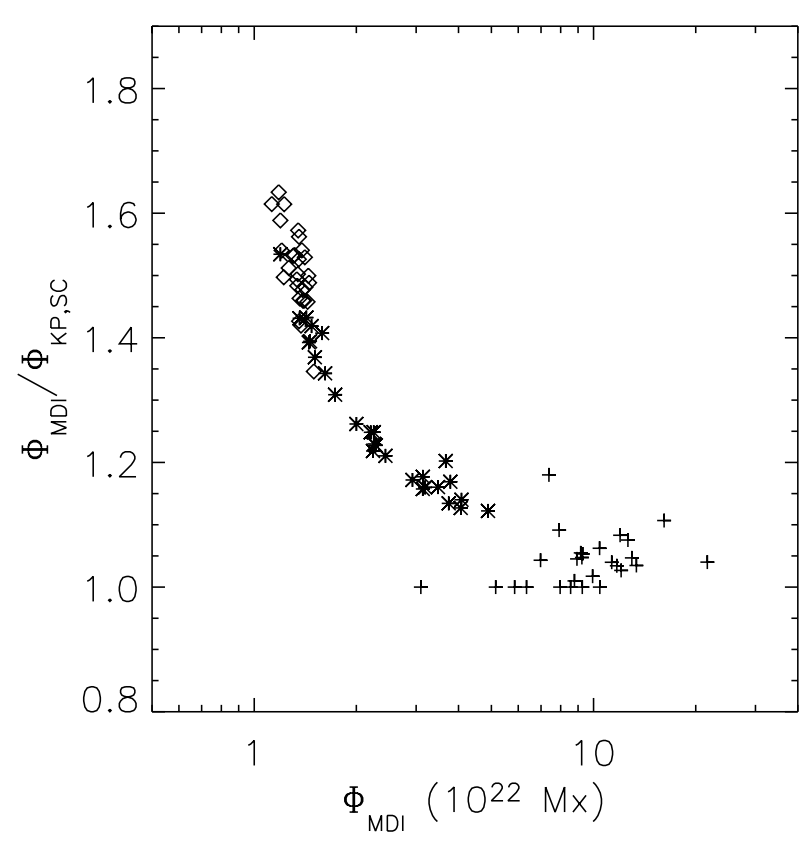

Fig. 2. Dependence of the $\Phi_{\mathrm{MDI}} / \Phi_{\mathrm{KP}}$, SC ratio on the total magnetic flux within a longitude band (see text for details). The noise threshold has been set at $1 \sigma$. Asterisks: total flux; plusses: active regions only; diamonds: quiet Sun only.

for active regions all lie close to 1 , since they contain unipolar patches that usually are bigger than a pixel in the KP synoptic chart. The $\Phi_{\mathrm{MDI}} / \Phi_{\mathrm{KP}, \mathrm{SC}}$ ratios grow quickly with decreasing magnetic flux - the effect of the resolution becomes more and more important with decreasing activity level, as mixed polarity fields play an increasing role. For the most quiet regions, $\Phi_{\mathrm{MDI}} / \Phi_{\mathrm{KP}, \mathrm{SC}}$ reaches $1.6-1.65$ with the $1 \sigma$ noise threshold. Interestingly, the quiet Sun and total flux points appear to lie on the same curve.

Since with $3 \sigma$ almost all the noise must have been eliminated, the 56-min average at $3 \sigma$ provides the absolute lower limit to the $\Phi_{\mathrm{MDI}} / \Phi_{\mathrm{KP}}$, SC ratio: at least $20 \%$ of the quiet-Sun flux is still hidden at the KP synoptic chart resolution (Table 1 and Fig. 3). This limit, however, appears to be too conservative, and the real ratio is likely to lie between those obtained for the 56-min averages at $1 \sigma$ and $2 \sigma$. This is indicated by a comparison of fluxes obtained for the synoptic maps with different integration time (or noise level) and noise threshold. At $1 \sigma$, the flux $\Phi_{\mathrm{MDI}}$ decreases from 1-min to 56-min averages, suggesting that there is still a lot of noise above $1 \sigma$ in $1-$ and 5 -min data, which goes down with integration time. Already at $2 \sigma$ the situation reverses: the flux increases with integration time, which implies that now we see more and more weak magnetic features as the integration time is increased and this effect overrides the decrease in the apparent signal due to lower noise. In Fig. 3, the quiet-Sun ratios $\Phi_{\mathrm{MDI}} / \Phi_{\mathrm{KP}}$, SC obtained with different thresholds are shown versus the noise level of the magnetograms. Linear extrapolations to $0 \mathrm{G}$ (infinitely long integration) shown by the dashed lines of the curves representing cutoffs at $1 \sigma$ and $2 \sigma$ yield a very similar value of $1.34-1.35$, while an extrapolation of the $3 \sigma$ curve provides a somewhat lower ratio of 1.26. Figure 3 thus implies that the threshold at which the noise that we keep is just outweighed by the weak real signal lost within the noise lies between the $1 \sigma$ and $2 \sigma$ cutoffs.

We believe that the ratios deduced by these extrapolations to $0 \mathrm{G}$, being based on $20 \mathrm{~min}$ and 56 min magnetograms, are too conservative, since an increasing fraction of the signal is lost also in the original resolution MDI magnetograms due to the motion and evolution of features as the integration time increases. We believe that it is this effect which is responsible for the convex shape of the curves outlined by the solid lines. The dotted lines represent linear extrapolations to $\sigma=0 \mathrm{G}$ from the $\Phi_{\mathrm{MDI}} / \Phi_{\mathrm{KP}}$, SC values obtained for 20 -min and 5-min integrations. In this case we obtain $\Phi_{\mathrm{MDI}} / \Phi_{\mathrm{KP}, \mathrm{SC}}(\sigma=0)$ values of $1.45,1.41$ and 1.3 with cutoffs at $1 \sigma, 2 \sigma$ and $3 \sigma$, respectively.

In the next section another, independent method is used to compare different thresholds. 


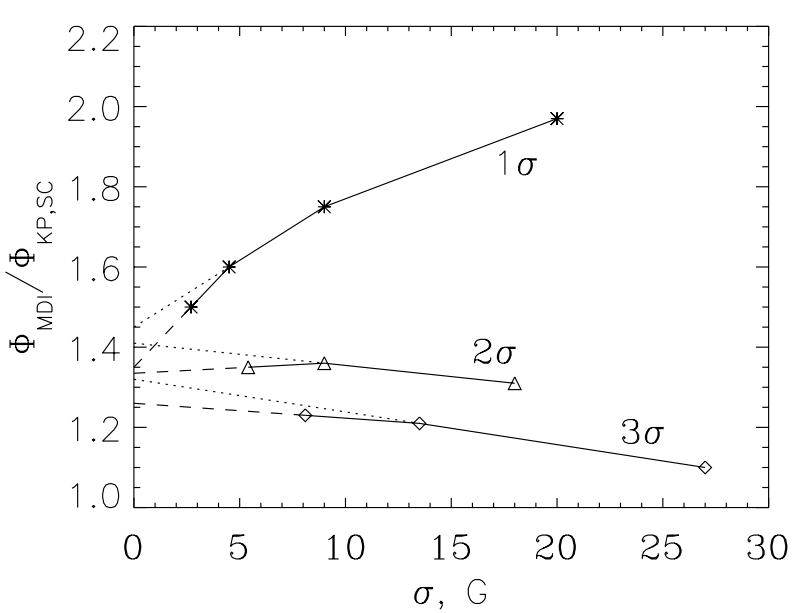

Fig. 3. Dependence of the $\Phi_{\mathrm{MDI}} / \Phi_{\mathrm{KP}}$, sC ratio on the noise level (i.e. total integration time) of magnetograms with cutoffs at $1 \sigma, 2 \sigma$ and $3 \sigma$. Plotted are the results for the quiet-Sun component. Dashed lines are linear extrapolations to the " 0 "-noise level corresponding to an infinitely long integration. Dotted lines are linear extrapolations from the lines joining the values obtained from 5 min and 20 min magnetograms.

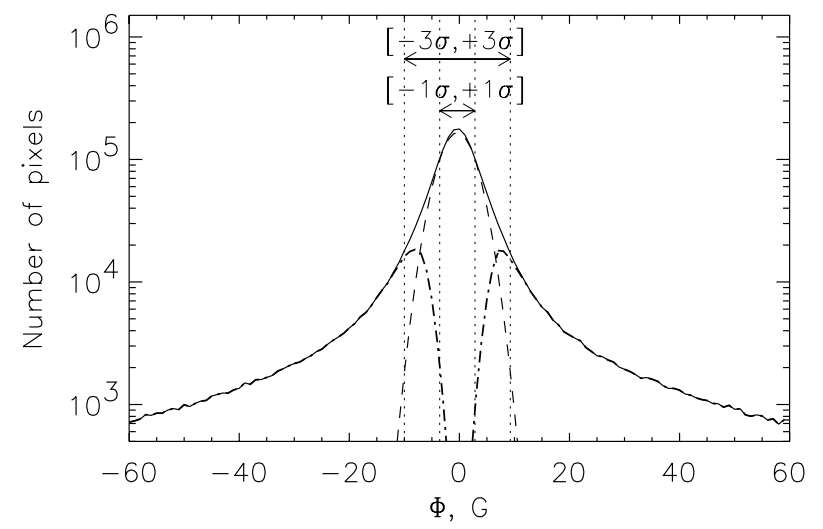

Fig. 4. Histogram (solid) of the magnetic signal $\Phi$ for the CR 1975 synoptic chart (56-min average). Dashed curve: a Gaussian fit representing the noise distribution (see text); dot-dashed curve: difference between the histogram of the total signal and the Gaussian noise distribution. Vertical dotted lines and arrows show the $\pm 1 \sigma$ and $\pm 3 \sigma$ intervals, where $\sigma$ is the width parameter of the Gaussian.

\subsection{Magnetogram signal hidden in the noise}

Let us now estimate the amount of weak signal which may still be hidden in the noise. We use the CR 1975 synoptic chart obtained from 56-min averages and construct a histogram of the magnetic signal (again for the total flux, quiet Sun and active regions individually). The core of the histogram for the total magnetic flux is shown in Fig. 4 (solid line). We have only included pixels with $\mu>0.8$. Since noise is randomly distributed over the pixels, it is expected to have a Gaussian distribution centred at $0 \mathrm{G}$. The histogram indeed appears to be similar to a Gaussian in its central part.

We now assume that magnetic signal in the core of the histogram is almost completely due to noise. To represent this noise, we fit a Gaussian, $\mathcal{G}$, to the histogram, $\mathcal{H}$, in such a way that it never exceeds $\mathcal{H}$, since the noise cannot exceed the total registered signal. This Gaussian is represented by the dashed line in Fig. 4. Now, the difference between $\mathcal{H}$ and $\mathcal{G}$ (dot-dashed curve) gives the distribution of the real solar signal and its integral over all pixels yields an estimate of the actual magnetic flux.

Most probably, a part of the signal falling within the fitted Gaussian is also real. One indication for this is that the value of $\sigma$ we obtain as the half width at half maximum of $\mathcal{G}$ is somewhat higher $(3.2 \mathrm{G})$ than the expected value for 56 -min averages $(\$ 20 / \sqrt{5} 6 \approx 2.7 \mathrm{G})$. Note, that for 1-min magnetograms our fitted Gaussians indeed give $\sigma \lesssim 20 \mathrm{G}$. Therefore, the excess width of the Gaussian fitted to the 56-min integration histogram must be due to true magnetic signal unless a source of significant systematic error is present in the data (such as remaining fixed pattern noise). Furthermore, we make the conservative assumption that at small values of $\Phi$ only noise is present, implying that the Gaussian has the same amplitude as the distribution around $\Phi=0$, i.e. $\mathcal{G} \approx \mathcal{H}(\Phi=0)$. This leads to the very steep and probably artificial decrease of the dot-dashed curve in Fig. 4 near $\Phi=0$. Therefore, the magnetic flux we find in this way represents a lower limit only. Instead of fitting a Gaussian, we have also used the somewhat narrower Gaussian with the nominal width of $2.7 \mathrm{G}$, scaled such that it nowhere exceeds the observed distribution. In the following we concentrate on the results obtained with the fitted Gaussian since they are more conservative.

In Table 2 we compare the total amount of flux in all pixels at $\mu>0.8$ deduced from $\mathcal{H}-\mathcal{G}$ with the flux $\Phi_{\mathrm{MDI}}(\mu>0.8)$ obtained by setting a threshold, $\Theta$, as described in Sect. 3.1. In Cols. 2-4 the ratios of the fluxes deduced from the techniques described in Sects. 3.1 and 3.2 are listed. The ratio is close to unity for $1 \sigma$ and steadily increases for larger thresholds. This is not surprising and only says that we are missing increasing amounts of magnetic flux when going to a higher threshold. Also not surprising is the fact that the ratio $\Phi(\mathcal{H}-\mathcal{G}) / \Phi_{\mathrm{MDI}}(\Theta)$ increases most rapidly for the quiet Sun, where the average field is weakest. We expect that the hidden flux indicated by $\Phi(\mathcal{H}-\mathcal{G}) / \Phi_{\mathrm{MDI}}(\Theta)>1$ is due to weak network and possibly intranetwork fields. Table 2 implies that although we indeed count some noise as the solar magnetic flux when taking $1 \sigma$ for the noise threshold, this is almost exactly offset by the magnetic flux masked by the noise. The fact that $\Phi(\mathcal{H}-\mathcal{G}) / \Phi_{\mathrm{MDI}}(1 \sigma) \approx$ 0.97 appears to imply that a slightly higher cutoff may be appropriate, but we remind the reader that the ratios in Table 2 are lower limits. We stress here that not all the points lying above $1 \sigma$ are real signal; only the amount of flux hidden below $1 \sigma$ is approximately equal to the contribution from noise above $1 \sigma$. Already at $\Theta=1.5 \sigma$ we start loosing signal, which is in good agreement with the results obtained independently in Sect. 3.1 (Fig. 3). With the $2 \sigma$ cut-off we have lost at least $15 \%$ of the actual flux in the quiet Sun and more than $30 \%$ with $3 \sigma$.

In Col. 5 of Table 2 we list the ratio of the flux deduced from the MDI synoptic chart compared with the synoptic chart at Kitt Peak resolution, $\Phi_{\mathrm{MDI}} / \Phi_{\mathrm{KP}}$, SC, for the 56-min averages (the numbers are partly repeated from Table 1). In order to obtain an improved estimate of the flux missed in KP synoptic charts we need to multiply $\Phi(\mathcal{H}-\mathcal{G}) / \Phi_{\mathrm{MDI}}$ (which is an indicator of the fraction of the flux hidden in the noise of MDI 
Table 2. Ratios (Cols. 2-4) between the magnetic flux of the CR 1975 synoptic map (56-min) obtained by two different methods, $\Phi_{\mathrm{MDI}}$ for different thresholds, $\Theta$, (given in Col. 1), and $\Phi(\mathcal{H}-\mathcal{G})$ (see Sects. 3.1 and 3.2, respectively, for descriptions of the two techniques). The 5th column lists the $\Phi_{\mathrm{MDI}} / \Phi_{\mathrm{KP}, \mathrm{SC}}$ ratios for the quiet Sun found in Sect. 3.1 (Table 1) and the last column is the product of Cols. 4 and 5.

\begin{tabular}{cccccc}
\hline \hline 1 & 2 & 3 & 4 & 5 & 6 \\
$\Theta$ & & $\frac{\Phi(\mathcal{H}-\mathcal{G})}{\Phi_{\mathrm{MDI}}}$ & & $\frac{\Phi_{\mathrm{MDI}}}{\Phi_{\mathrm{KP}, \mathrm{SC}}}$ & $\frac{\Phi(\mathcal{H}-\mathcal{G})}{\Phi_{\mathrm{KP}, \mathrm{SC}}}$ \\
& $\mathrm{AR}+\mathrm{QS}$ & $\mathrm{AR}$ & $\mathrm{QS}$ & $\mathrm{QS}$ & $\mathrm{QS}$ \\
\hline $1 \sigma$ & 0.99 & 1.00 & 0.97 & 1.50 & 1.46 \\
$1.5 \sigma$ & 1.02 & 1.00 & 1.06 & 1.43 & 1.52 \\
$2 \sigma$ & 1.05 & 1.01 & 1.15 & 1.35 & 1.55 \\
$3 \sigma$ & 1.10 & 1.02 & 1.32 & 1.23 & 1.62 \\
\hline
\end{tabular}

magnetograms) with $\Phi_{\mathrm{MDI}} / \Phi_{\mathrm{KP}, \mathrm{SC}}$. This product, listed in the last column of Table 2, gives our refined estimates of the total flux found with the full-disc MDI data with respect to the flux obtained with the KP synoptic chart resolution. Encouragingly, these final numbers depend only weakly on the threshold $\Theta$. The numbers imply that at least $1 / 3$ of the quiet-Sun magnetic flux went uncounted in KP synoptic charts.

\subsection{High-resolution MDI magnetograms}

In this section we consider the possibility that even the spatial resolution of the MDI full disc magnetograms is insufficient to avoid apparent cancellation of some flux. To check this to the extent possible, we have also examined $14 \mathrm{MDI}$ high-resolution (HR) magnetograms obtained at different activity levels, from May, 1996 to July, 2001. We take 20-min averages with a mean noise level of $1 \sigma \approx 4.5 \mathrm{G}$ (see Sect. 2 for the reasoning behind this choice).

We first study the dependence of the total measured flux on the size of a pixel formed by binning together different numbers of pixels of the high-resolution magnetograms. Figure 5 shows how the total flux (normalized to the flux for the MDI HR mode, i.e. for unbinned pixels) depends on the number of HR pixels that were combined. Of the 14 analysed HR magnetograms, 4 included active areas; these are represented by filled symbols. For reference, three of the analysed magnetograms at different activity levels are exhibited in Fig. 6: the top panels show the original MDI HR data whereas the bottom panels are for the resolution reduced to that of the KP synoptic charts. The magnetograms displayed in Figs. $6 \mathrm{a}-\mathrm{c}$ are represented respectively in Fig. 5 by open squares, filled triangles and filled diamonds. Based on the results of the previous section, we used here $1 \sigma$ as a threshold. The dependence on spatial resolution, particularly strong for the quiet Sun, is clearly seen. For the quiet Sun, fluxes above $1 \sigma$ measured for the MDI full disc resolution are about a factor 1.7-1.8 higher than for the KP synoptic chart resolution.

This value is somewhat higher than those given in Table 1. The reason for this mainly lies in the different geometry of the

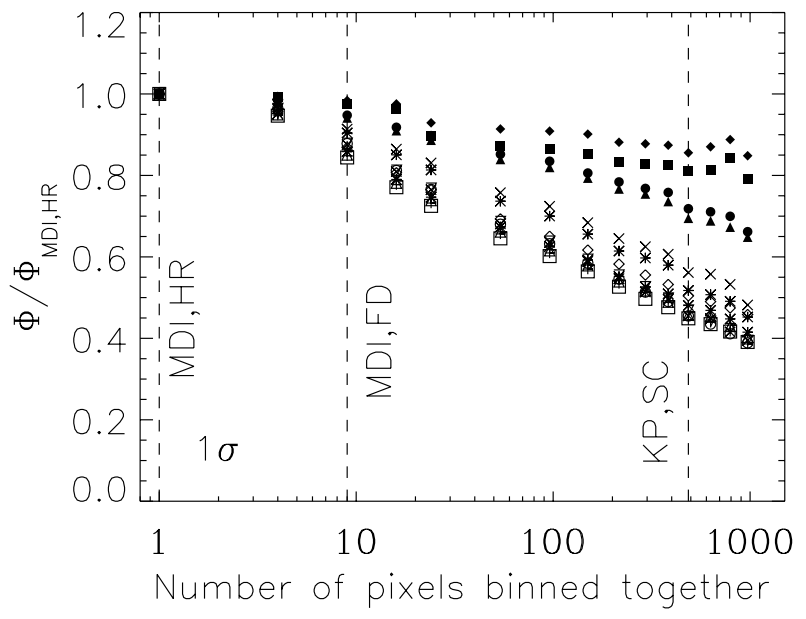

Fig. 5. Dependence of the measured magnetic flux on the size of a bin (or pixel) for 14 analysed MDI high-resolution (HR) magnetograms. Fluxes are normalized to the flux corresponding to the MDI HR mode. Signal below $1 \sigma$ is assumed to be noise and has been removed. Filled symbols are used for magnetograms containing active areas. The dashed lines correspond to the resolution of the high resolution (MDI, HR) and the full-disc MDI magnetograms (MDI, FD) as well as the Kitt Peak synoptic charts (KP, SC).

synoptic charts, which sample also the polar regions and the HR magnetograms, which are restricted to solar disc centre. When preparing the synoptic charts and the HR magnetograms for analysis, we first exclude pixels below the noise threshold and then divide the remaining signal by $\mu$ (see Sect. 2 ). In this way, much more weak signal is eliminated in the polar regions than at disc centre. However, this weak signal is most susceptible to cancellation.

Extrapolating linearly in Fig. 5 to even higher resolution we estimate that another $5-10 \%$ of the magnetic flux may be lost even in the $0.6^{\prime \prime}$ pixels of the HR mode. Signs of saturation of the curves at the highest resolution make this estimate uncertain. At the same time significantly more flux may be hidden in the form of weak turbulent fields whose presence is indicated by Hanle-effect measurements (Stenflo et al. 1998; Berdyugina \& Fluri 2004), so that the $5-10 \%$ quoted above is probably conservative.

In order to compare more accurately the high-resolution MDI magnetograms with those having the KP synoptic chart resolution, we plot in Fig. 7 ratios of the fluxes at the two resolutions as a function of the total flux for a threshold of 1,2 and $3 \sigma$. Every symbol represents one magnetogram. The absolute lower limit for the obtained ratio, i.e. when only flux above $3 \sigma$ is taken into consideration, during activity minimum is about 1.3. The real ratio, which we estimate corresponds to a threshold close to $1 \sigma$, is likely to be between 2 and 2.2.

\section{Conclusions}

Accurate estimates of the amount, relative distribution (quiet Sun vs. active regions) and evolution of the solar total magnetic flux are essential for a quantitative assessment of the secular change in the solar magnetic flux and irradiance (Solanki et al. 2002; Solanki 2002) and therefore have an important 

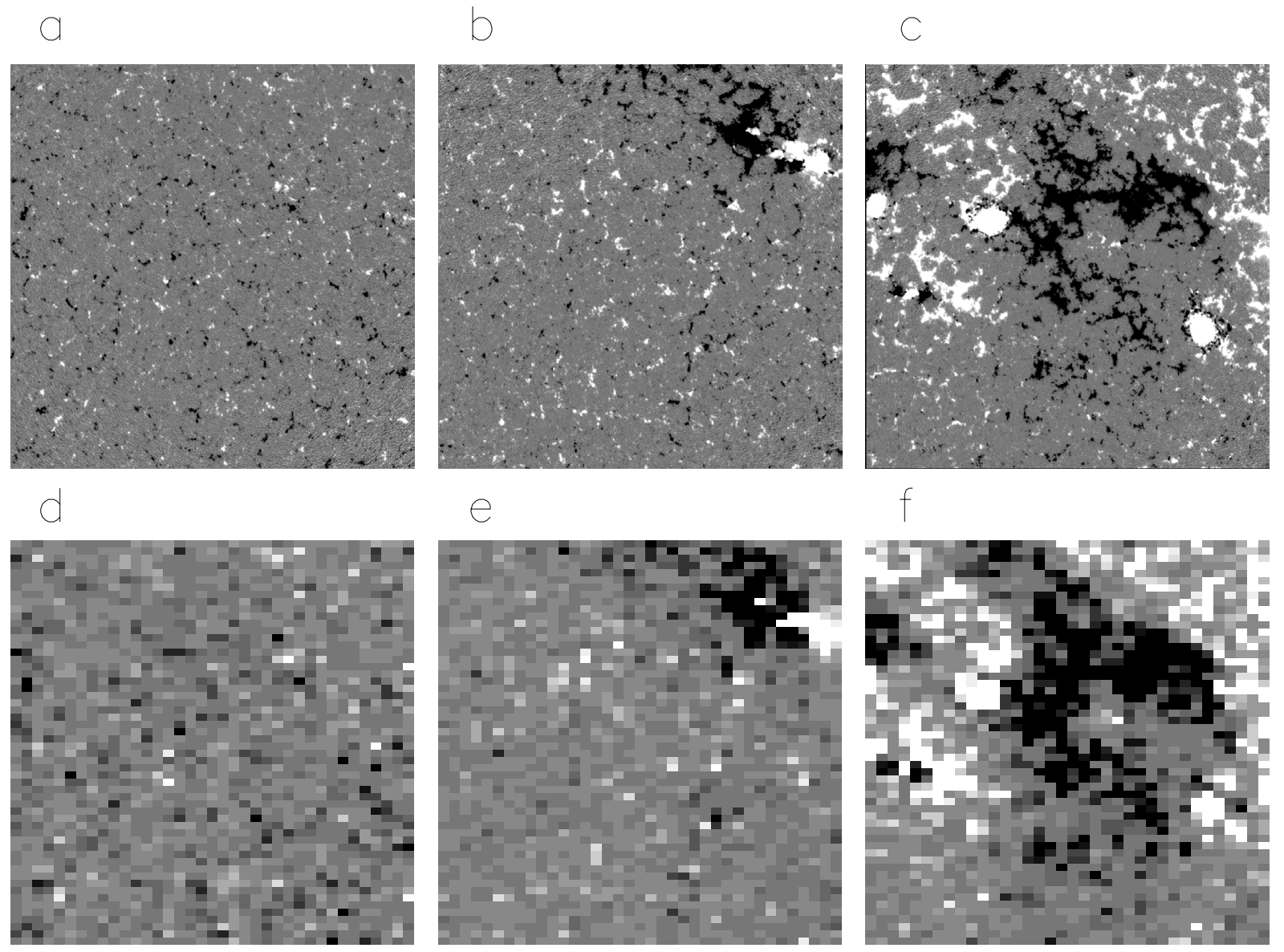

Fig. 6. Examples of high-resolution and degraded magnetograms. Top: MDI high-resolution mode; bottom: Kitt Peak Synoptic Chart resolution. a) and d): quiet Sun (8 May 1996, shown by open squares in Fig. 5). b) and e): small active region (6 November 1997, filled triangles in Fig. 5). c) and f): active Sun (17 July 2001, filled diamonds in Fig. 5). Dark and light mark negative and positive polarities, respectively, and grey indicates zero flux.

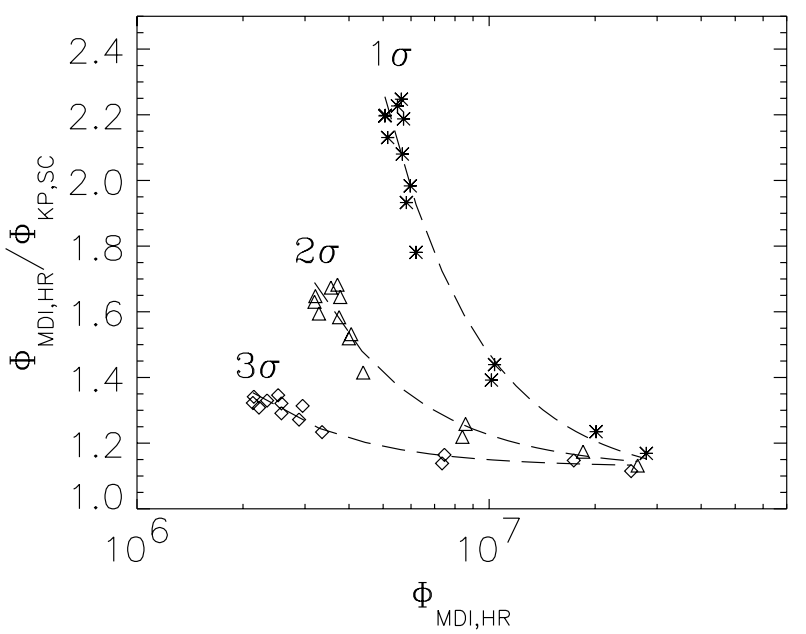

Fig. 7. Ratio of magnetic fluxes obtained for the MDI, HR and KP synoptic chart resolutions as a function of the total flux. Three cases, with the threshold being $1 \sigma, 2 \sigma$ and $3 \sigma$, respectively, are shown.

bearing on studies of Sun-climate relations. KP synoptic charts are a widely used resource, also to estimate the Sun's total flux. Their spatial resolution is, however, insufficient to resolve elements of the magnetic network. We use MDI full-disc and high-resolution magnetograms to estimate the total magnetic flux present on the Sun and how much of it remains visible at the resolution of KP synoptic charts. The analysis has been carried out separately for the Sun as a whole, quiet Sun regions and active regions. Whereas for active regions, the effect of the spatial resolution is rather unimportant, significant magnetic flux contained in small-scale magnetic elements in the quiet Sun goes uncounted because opposite polarities are present within a single resolution element of the Kitt Peak synoptic charts. This result is consistently returned for different analysis techniques.

Compared to the full-disc resolution of MDI, at least $1 / 3$ of the quiet-Sun flux escapes notice in KP synoptic charts. This means that the ratio $\Phi_{\mathrm{AR}} / \Phi_{\mathrm{QS}}$ is, in fact, distinctly lower than found from the KP synoptic charts.

However, even with this resolution there is still some flux going unnoticed, which becomes evident from the analysis of MDI magnetograms obtained in the HR mode. Employment of these high-resolution data leads to a factor of 2.0-2.2 with respect to the KP synoptic charts. When taken together, the results presented here suggest that only approximately half of the magnetic flux in the quiet Sun is visible in Kitt Peak synoptic charts. This can be combined with the result found by Harvey (1994) that at maximum the flux in active regions is 2-3 times that in the quiet Sun. It then implies that the magnetic flux 
present on the solar surface at activity minima is approximately comparable to the total flux in active regions at strong maxima of activity such as reached during cycles 21 and 22 . This corresponds to approximately twice as much total flux on the Sun during recent maxima of solar activity compared to recent minima. Interestingly, this result was independently returned by the models of Solanki et al. (2002) for the evolution of the Sun's total magnetic flux, while Schrijver et al. (2002) obtain a result intermediate to that obtained by Harvey (1994) and what we get in this paper.

Acknowledgements. We are grateful to M. Fligge for initial work from which the analysis presented here has greatly profited. We thank R. Bush for useful discussions on the detailed behaviour of MDI and H. Uitenbroek for helpful comments on the manuscript.

\section{References}

Berdyugina, S. V., \& Fluri, D. 2004, A\&A, 417, 775

Fligge, M., Solanki, S. K., Meunier, N., \& Unruh, Y. C. 2000a, ESA-SP, 463, 117

Fligge, M., Solanki, S. K., \& Unruh, Y. C. 2000b, A\&A, 353, 380
Fröhlich, C. 2000, Sp. Sci. Rev., 94, 15

Harvey, K. L. 1994, in The Sun as a Variable Star: Solar and Stellar Irradiance Variations, ed. J. M. Pap, C. Fröhlich, H. S. Hudson, \& S. K. Solanki (Cambridge: Cambridge Univ. Press), IAU Coll., 143, 217

Krivova, N. A., Solanki, S. K., Fligge, M., \& Unruh, Y. C. 2003, A\&A, 399, L1

Ortiz, A., Solanki, S. K., Domingo, V., Fligge, M., \& Sanahuja, B. 2002, A\&A, 388, 1036

Scherrer, P. H., Bogart, R. S., Bush, R. I., et al. 1995, Sol. Phys., 162, 129

Schrijver, C. J., DeRosa, M. L., \& Title, A. M. 2002, ApJ, 577, 1006

Solanki, S. K. 2002, in From Solar Min to Max: Half a Solar Cycle with SOHO, ESA-SP, 508, 173

Solanki, S. K., Schüssler, M., \& Fligge, M. 2000, Nature, 408, 445

Solanki, S. K., Schüssler, M., \& Fligge, M. 2002, A\&A, 383, 706

Stenflo, J. O., Keller, C. U., \& Gandorfer, A. 1998, A\&A, 329, 319

Willson, R. C., \& Hudson, H. S. 1988, Nature, 332, 810

Willson, R. C., \& Hudson, H. S. 1991, Nature, 351, 42

Wilson, A. 2000, The solar cycle and terrestrial climate, Solar and space weather Euroconference, ESA-SP, 463 (Noordwijk, Netherlands: ESA Publications Division) 